\title{
A STUDY OF THE EFFECTS OF AMLODIPINE AND CILNIDIPINE ON HAEMODYNAMIC PARAMETERS AND RENOPROTECTION IN HYPERTENSIVE SUBJECTS WITH PROTEINURIA
}

\author{
Laxme Gowda1, Ramesh S. S2
}

${ }^{1}$ Associate Professor, Department of Medicine, Mysore Medical College and Research Institute, Mysore. ${ }^{2}$ Associate Professor, Department of Medicine, Mysore Medical College and Research Institute, Mysore.

\section{ABSTRACT}

\section{BACKGROUND}

Hypertension with chronic kidney disease is a widely prevalent public health concern. Calcium channel blockers are a commonly used class of drugs for the treatment of hypertension. L-type calcium channel blockers like amlodipine cause a reflex sympathetic overactivity, which predisposes to increased cardiovascular morbidity and mortality. Also, the effect of L-type CCBs on urinary protein excretion is uncertain. Cilnidipine is a novel CCB with a dual $\mathrm{L} / \mathrm{N}$-type calcium channel blocking property, thus favouring additional renal and cardiovascular protection.

The objective of this study is to evaluate the effects and their linearity across the timeframe of amlodipine and cilnidipine in hypertensive subjects with proteinuria on heart rate, blood pressure, lipid profile and proteinuria.

\section{MATERIALS AND METHODS}

After Institutional Ethical Committee approval, a prospective, randomised and open-label study was carried out on hypertensive subjects with proteinuria attending the General Medicine OPD in K. R. Hospital, Mysore. Sixty subjects satisfying the inclusion and exclusion criteria were included in the study. Heart rate, blood pressure, lipid profile (TC, LDL, HDL, TG) and Urine Protein-toCreatinine Ratio (UPCR) were measured at baseline. Blood pressure and heart rate were monitored at weekly intervals until the end of 12 weeks. While lipid profile was reassessed at 6 weeks and 12 weeks, UPCR was reassessed at the end of 12 weeks. Descriptive statistics, independent sample ' $t$ ' test, repeated measure ANOVA and Cramer's $V$ test were used to analyse the results.

\section{RESULTS}

Demographic profile was well matched in both the groups. In the Amlodipine group, the heart rate was significantly higher than that before treatment, whereas subjects in the cilnidipine group had a significantly lower heart rate when compared to baseline ( $\mathrm{p}$ $<0.05$ ). There was no significant difference in mean SBP and mean DBP values, either within each group or between the two groups. Also, the UPCR was significantly decreased in the cilnidipine group as opposed to the amlodipine group where it was significantly increased, thereby resulting in a significant intergroup difference $(p<0.05)$. However, neither of the drugs caused a significant change in the lipid parameters and the intergroup difference was also statistically insignificant.

\section{CONCLUSION}

Cilnidipine is thus, a better alternative in hypertensive patients with proteinuria due to its cardioprotective and renoprotective actions.

\section{KEYWORDS}

Hypertension; Proteinuria; Heart Rate; Amlodipine; Cilnidipine.

HOW TO CITE THIS ARTICLE: Gowda L, Ramesh SS. A study of the effects of amlodipine and cilnidipine on haemodynamic parameters and renoprotection in hypertensive subjects with proteinuria. J. Evolution Med. Dent. Sci. 2017;6(18):1430-1438, DOI: 10.14260/Jemds/2017/313

\section{BACKGROUND \\ Hypertension (HTN) is an enormous health problem and is one of the biggest health challenges in the 21 st century. The Global Burden of Disease study has reported HTN as the $4^{\text {th }}$ contributor to premature death in developed countries and the $7^{\text {th }}$ in the developing countries. Although the condition is common, readily detectable and easily treatable, it is usually asymptomatic and often leads to lethal complications if left untreated. ${ }^{1}$ Hypertension is a risk factor for the development}

Financial or Other, Competing Interest: None.

Submission 02-02-2017, Peer Review 17-02-2017,

Acceptance 21-02-2017, Published 02-03-2017.

Corresponding Author:

Laxme Gowda,

Associate Professor,

Department of Medicine,

Mysore Medical College and Research Institute,

Mysore.

E-mail: laxmegowda@gmail.com

DOI: $10.14260 /$ jemds $/ 2017 / 313$

\section{(c) $($ ) $९$}

of cardiovascular diseases such as Coronary Heart Disease (CHD), Congestive Heart Failure (CHF), ischaemic and haemorrhagic stroke, renal failure and peripheral arterial disease. ${ }^{2,3}$

The ultimate objective of antihypertensive treatment is to prevent cerebrovascular or cardiovascular disease. 4 Advances in the diagnosis and treatment of hypertension have played a major role in recent dramatic declines in coronary heart disease and stroke mortality in industrialised countries. 5 Treatment for hypertensive patients includes both non-pharmacologic (Lifestyle changes) and pharmacologic therapy to lower blood pressure and prevent cardiovascular events such as a heart attack. Implementation of lifestyle interventions should be used throughout the management of all patients with high blood pressure. ${ }^{6}$

Several classes of antihypertensive drugs have been in clinical use. ${ }^{7}$ The choice of drugs is influenced by the age, ethnicity/race, other conditions (eg, diabetes and coronary disease) associated with hypertension and pregnancy. ${ }^{8}$ 
Thiazide and Thiazide-like Diuretics, Angiotensin Converting Enzyme (ACE) inhibitors, Angiotensin Receptor Blockers (ARBs) and Calcium Channel Blockers (CCBs) are the first line agents, whereas beta blockers, $\alpha$ blockers, aldosterone antagonists and direct renin inhibitors form the second line therapeutic options. Vasodilators including minoxidil and hydralazine should be used as a last-line option to treat HTN. ${ }^{6}$

Calcium channel blockers were introduced 25 years ago as coronary vasodilators and have since achieved notable recognition in the treatment of arterial hypertension. ${ }^{9}$ Multiple types of voltage-gated $\mathrm{Ca}^{2+}$ channels were first distinguished by voltage- and time-dependence of channel gating, single channel conductance and pharmacology. Presently, they are classified into T-, L-, N-, P/Q- and R-type. 10 In the cardiovascular system, L-type $\mathrm{Ca}^{2+}$ channels are predominantly expressed in the heart and vessels, which regulate cardiac contractility, sinus nodal function and vascular tone. Thus, the channel has been recognised as a pharmacological target for the treatment of cardiovascular disease. ${ }^{11}$

With the only exception of renal diseases in which ReninAngiotensin System (RAS) blocking agents should be preferred; CCBs are now recommended as first-line therapy in all stages of hypertension independent of age, gender, race, and other comorbidities. Beyond evidence in favour of cardiovascular and renal protection, clinical trials have also demonstrated a better tolerability profile and favourable metabolic properties by RAS-inhibiting drugs, especially ARBs and by CCBs compared to $\beta$-blockers and diuretics, thus promoting the use of these drug classes, both in monotherapy and in combination therapies for hypertension management and control.12

Diabetic and black hypertensive patients with low renin levels, salt-sensitive and volume-expanded are more often responsive to a CCB than to an ACE inhibitor or a $\beta$ blocker. Also, the response to increasing the dose of a CCB is generally that of significant additional BP reduction, which separates this drug class from both ACE inhibitors and ARBs. ${ }^{13}$ Indeed, monotherapy based on CCBs has demonstrated to provide sustained antihypertensive efficacy and to reduce cardiovascular and renal complications compared to other antihypertensive drug classes. ${ }^{12}$ Calcium channel blockers are effective as single-drug therapy in approximately $60 \%$ of patients in all demographic groups and all grades of Hypertension. ${ }^{2}$ However, the reduction of blood pressure associated with these agents is associated with reflex sympathetic overactivity, which leads to increased cardiovascular morbidity and mortality. ${ }^{14}$

HTN has been reported to occur in $85 \%$ to $95 \%$ of patients with CKD (Stages 3 - 5). The relationship between HTN and CKD is cyclic in nature. Uncontrolled HTN is a risk factor for developing CKD and is associated with a more rapid progression of CKD15. Increased blood pressure has a major role in the development of proteinuria in patients with either diabetic or non-diabetic kidney disease. Proteinuria is a manifestation of overt nephropathy, which increases the risk of cardiovascular diseases and causes rapid deterioration of kidney function. ${ }^{3}$ Multiple guidelines discuss the importance of lowering Blood Pressure (BP) to slow the progression of renal disease and reduce cardiovascular morbidity and mortality. 15
Drugs interfering with the renin-angiotensin system, i.e. angiotensin converting enzyme inhibitors and angiotensin receptor blockers should be used as first-line antihypertensive therapy in patients with proteinuria, because they seem to have a blood pressure-independent antiproteinuric effect. ${ }^{3}$ Calcium channel blockers form an effective combination therapy with Angiotensin Converting Enzyme (ACE) inhibitors or Angiotensin Receptor Blockers (ARBs) in hypertensive patients with preserved renal function or with CKD. ${ }^{16}$ However, CCBs are not always able to protect against kidney injury due to the presence of L-type calcium channels at the afferent but not efferent arterioles, which increases the glomerular pressure. ${ }^{17}$

Once-daily amlodipine provides a favourable side effect profile without adversely affecting neurohumoral or metabolic parameters, thereby providing safe and reliable 24-hour therapy for patients with coronary heart disease and/or arterial hypertension. ${ }^{9}$ However, such pharmacokinetic and pharmacodynamic improvements do not always evade the sympathetic reflex induced by the hypotensive action of L-type Ca2+ channel blockers. ${ }^{11}$ Also, the renoprotective effect of L-type CCBs like amlodipine is very uncertain. ${ }^{17}$

Cilnidipine is a novel dihydropyridine derivative with a dual L/N-type calcium channel blocking property. The antihypertensive effect of cilnidipine has been demonstrated in hypertensive patients and also in patients with severe hypertension or with complications such as chronic kidney disease, cerebrovascular disease and diabetes. In individuals with diabetes, Cilnidipine also improves lipid profile ${ }^{18}$ and insulin resistance. ${ }^{19}$

$\mathrm{N}$-type $\mathrm{Ca}^{2+}$ channels are localised at the nerve endings in the sympathetic and central nervous systems, which regulate the release of neurotransmitters. ${ }^{8}$ The anti-sympathetic action of cilnidipine has been demonstrated in several experimental studies and is thought to be due to blockade of N-type calcium channels. ${ }^{19}$ Studies have shown that Cilnidipine, a L/N-type calcium channel blocker not only blocks reflex sympathetic stimulation but also reduces proteinuria, thus providing additional renal and cardiovascular protection.4,14

Hence, the present study was undertaken to evaluate the renoprotective and cardiovascular benefits of cilnidipine in comparison to amlodipine.

\section{Objectives}

To evaluate the effects and their linearity across the timeframe of amlodipine and cilnidipine in hypertensive subjects with proteinuria on the following parameters -

1. Heart rate.

2. Blood pressure.

3. Lipid profile (Total cholesterol, total triglycerides, LDL, HDL).

4. Proteinuria.

\section{MATERIALS AND METHODS}

This prospective study was done to compare the efficacy of amlodipine and cilnidipine among hypertensive subjects with proteinuria. 


\section{Source of Data}

Primary source of information with observation technique was adopted on hypertensive subjects with proteinuria attending General Medicine Department of K. R. Hospital, Mysore, during January 2015 to December 2015.

\section{Study Design}

Randomised, parallel group, open label, single-centre study.

\section{Study Period}

January 2015 - June 2016.

\section{Sample Design}

Purposive sampling technique.

\section{Sample Size}

Using estimation technique with prevalence of hypertensive subjects with proteinuria as $4-16 \%$, effect size $10 \%$ and level of significance as $5 \%$, sample size was calculated to be 15 and 53 for $4 \%$ and $16 \%$ prevalence respectively. We decided to go with 60 subjects divided into two groups of 30 each.

\section{Inclusion Criteria}

- $\quad$ Age $>/-40$ years.

- Both sexes.

- Hypertension with coexisting proteinuria.

- Subjects who give informed consent.

\section{Exclusion Criteria}

- $\quad$ Subjects with SBP >/-180 mmHg and/or DBP >/- 110 mmHg prior to or during the washout period.

- Normotensive subjects with proteinuria.

- Hypertensive subjects on two or more antihypertensive medications.

- $\quad$ End-stage renal disease.

- Congestive heart failure.

- Heart block.

- Aortic stenosis.

- Pregnant and lactating women.

- Subjects on amlodipine/Cilnidipine/ACE inhibitors/ angiotensin receptor blockers within 30 days prior to their enrolment into the study.

\section{Method of Collection of Data}

After getting clearance from the Institutional Ethical Committee, hypertensive subjects attending medicine OPD in K. R. Hospital were screened for selection for the study. The subjects were well acquainted with the type of study and a written informed consent was taken.

\section{Screening Visit}

A complete medical history was taken and physical examination was conducted. Later, following tests were performed -

- $\quad$ ECG.

- Blood pressure - subjects having uncontrolled hypertension (SBP >/- $140 \mathrm{mmHg}$ and/or DBP >/- 90 $\mathrm{mmHg}$ at the screening visit were excluded from the study due to ethical considerations.
- Urine routine - only those subjects having urinary albumin $1+$ or more on dipstick analysis were considered for the study.

- $\quad$ FBS and PPBS.

- Serum creatinine and blood urea nitrogen.

\section{Echocardiography}

If history and/or physical examination suggestive of congestive heart failure or aortic stenosis.

Seventy-two subjects were screened, out of which 12 subjects were excluded due to non-fulfilment of inclusion or exclusion criteria. The remaining 60 subjects were asked to report back 3 days after the screening visit to record the baseline values.

\section{Following Parameters were recorded at Baseline}

- Heart rate.

- Measurement of heart rate: Using a finger probe pulse oximeter (Easy Care fingertip pulse oximeter).

- Blood pressure in sitting and standing position.

\section{Measurement of Blood Pressure}

Blood pressure was recorded in the arm, which showed a higher BP value during screening using a mercury sphygmomanometer with a cuff size of $12 \mathrm{~cm}$.

- For sitting blood pressure, the mean of three consecutive readings taken 30 to 60 s apart in the sitting position after a 5 min rest was considered.

- For recording standing blood pressure the subjects were first asked to lie down for 5 minutes, after which they were asked to stand. The blood pressure was recorded at 1 and 3 minutes after standing and the mean of these two readings was considered.

- It was made sure that subjects had not smoked or taken tea or coffee for half an hour before the blood pressure recording.

Lipid Profile (Total Cholesterol, Total Triglycerides, LDL, HDL)

- HDL and LDL: Assay based on a modified Polyvinyl Sulfonic Acid (PVS) and Polyethylene-Glycol Methyl Ether (PEGME) coupled with classic precipitation method.20,21

- Triglycerides: Assay done using the reagent, which is based on the method of Wako and the modification by McGowan et al and Fossati et al.22

- Cholesterol: The estimation of cholesterol was done using modified Roeschlau's method. ${ }^{23}$

Quantitative Estimation of Proteinuria - Urine ProteinCreatinine Ratio (UPCR)

The protein content in urine was measured by turbidimetric method in a spot urine sample using sulphosalicylic acid and sodium sulphate and the urinary creatinine concentration was measured by Jaffe's reaction. ${ }^{24}$ Urinary protein excretion in terms of $\mathrm{mg} / \mathrm{mg}$ of urinary creatinine was calculated to give the Urine Protein-Creatinine Ratio (UPCR). ${ }^{25}$

The enrolled subjects were randomised into 2 groups of 30 each using a computer generated random number table.

\section{Group A}

Received Tab. Amlodipine 5 - $10 \mathrm{mg} /$ day for 3 months. 


\section{Group B}

Received Tab. Cilnidipine 5 - $20 \mathrm{mg} /$ day for 3 months.

The dose of amlodipine and cilnidipine was titrated depending on the blood pressure control. If the goal SBP and/or DBP as per JNC-8 guidelines for management of hypertension [JNC-8a] was not reached at the end of one month of active treatment period, then those subjects were regarded as non-responders. The non-responders in Group A and Group B received increased dose of amlodipine or cilnidipine respectively for effective BP control. Otherwise, the same dose was continued throughout the study period.

Subjects were also informed about the known adverse effects of the respective drugs and were asked to report back anytime if necessary.

\section{Following Parameters were recorded at each Visit- 3 Weeks followup Visit}

1. Heart rate.

2. Blood pressure in sitting and standing position.

3. Adverse effects if any were noted and necessary measures were taken.

Subjects not attaining goal BP values at 3 weeks were followed up at 4 weeks to determine whether an increment in dose was warranted.

\section{Weeks followup Visit}

1. Heart rate.

2. Blood pressure in sitting and standing position.

3. Lipid profile (total cholesterol, total triglycerides, LDL, HDL).

4. Adverse effects if any were noted and necessary measures were taken.

\section{Weeks followup Visit}

1. Heart rate.

2. Blood pressure in sitting and standing position.

3. Lipid profile (total cholesterol, total triglycerides, LDL, HDL).

4. Quantitative estimation of proteinuria - urine proteincreatinine ratio.

5. Adverse effects if any were noted and necessary measures were taken.

Apart from this, blood pressure and heart rate were recorded at weekly intervals after the baseline visit until the completion of the evaluation period.

\section{Statistical Analysis}

Descriptive statistics and Cramer's V test was used to analyse the demographic variables. Repeated measure ANOVA was used to analyse the variation in each parameter from baseline till the end of 12 weeks. For intergroup comparison, independent sample ' $\mathrm{t}$ ' test was used. The entire data was analysed using Microsoft Excel and R software. A P value of < 0.05 was considered to be significant.

\section{RESULTS}

The increase in mean UPCR in the amlodipine group and the decrease in mean UPCR in the cilnidipine group are both statistically significant (Independent sample ' $\mathrm{t}$ ' test-
$P<0.001)$. Also, the intergroup difference in the change in mean UPCR from baseline was statistically significant at 12 weeks of treatment (Independent sample ' $t$ ' test - $p<0.001$ ).

The subjects in cilnidipine group had a significantly higher mean heart rate at baseline compared to the subjects in amlodipine group $(\mathrm{P}<0.049)$. The change in mean heart rate from baseline was significant at 3 weeks, 6 weeks and 12 weeks in both amlodipine group as well as cilnidipine group (ANOVA - $\mathrm{P}<0.001$ ). However, the intergroup difference in the change in mean heart rate from baseline was significant only at 6 weeks and 12 weeks (Independent sample ' $t$ ' test $\mathrm{p}<0.01$ ), but not at 3 weeks (Independent sample ' $\mathrm{t}$ ' test $\mathrm{p}>0.05$ ) of treatment with the study medications.

The change in mean sitting SBP from baseline was not significant at 3 weeks, 6 weeks and 12 weeks between the two groups (Independent sample ' $t$ ' test $-p>0.05$ ). Also, the variation in sitting SBP was not statistically significant in either of the groups. (ANOVA - $p>0.05$ ).

The change in mean sitting DBP from baseline was not significant at 3 weeks, 6 weeks and 12 weeks between the two groups (Independent sample ' $t$ ' test - $p>0.05$ ). Also, the variation in sitting DBP was not statistically significant in either of the groups. (ANOVA - $\mathrm{p}>0.05$ ).

The change in mean standing SBP from baseline was not significant at 3 weeks, 6 weeks and 12 weeks between the two groups (Independent sample ' $t$ ' test - $p>0.05$ ). Also, the variation in standing SBP was not statistically significant in either of the groups. (ANOVA - $p>0.05$ ).

The change in mean standing DBP from baseline was not significant at 3 weeks, 6 weeks and 12 weeks between the two groups (Independent sample ' $t$ ' test $-\mathrm{p}>0.05$ ). Also, the variation in standing DBP was not statistically significant in either of the groups. (ANOVA - $p>0.05$ ).

The mean total serum cholesterol shows an increasing trend in both the groups, but the increase is not significant (ANOVA - $p>0.05$ ). Also, the intergroup difference in terms of change in mean total serum cholesterol from baseline is not significant either at 6 weeks or at 12 weeks (Independent sample ' $\mathrm{t}$ ' test - $\mathrm{p}>0.05$ ).

The change in mean HDL levels is not significant in either group (ANOVA - $p>0.05$ ). Also, the intergroup difference in terms of mean change in HDL levels is insignificant (Independent sample ' $\mathrm{t}$ ' test $-\mathrm{p}>0.05$ ) at 6 weeks and 12 weeks.

While there is an increase in mean $\mathrm{TG}$ levels in comparison to baseline at 6 weeks and 12 weeks in both the groups, the difference is not statistically significant (ANOVA $\mathrm{p}>0.05$ ). Also, the intergroup difference in terms of change in mean LDL levels from baseline is statistically insignificant (Independent sample ' $\mathrm{t}$ ' test $-\mathrm{p}>0.05$ ) at 6 weeks and 12 weeks.

Though the mean LDL levels at the end of 12 weeks have decreased in comparison to baseline in both the groups, the difference over the 12 -week period is not statistically significant in both the groups (ANOVA - $p>0.05$ ). Also, the change in mean LDL levels from baseline is not statistically significant between the two groups at 6 weeks as well as 12 weeks (Independent sample ' $\mathrm{t}$ ' test $-\mathrm{p}>0.05$ ).

\section{Adverse Effects}

The adverse effect reported in the Group I after starting amlodipine were headache [6 (20\%)], dizziness [6 (20\%)], 
pedal oedema [5 (16.7\%)] and palpitation [4 (10\%)]. In Group II following cilnidipine therapy, the adverse effects noted were dizziness [8 (16.7\%)], headache [5 (16.7\%)] and pedal oedema [1 (3.3\%)]. The adverse effects noted in both the groups were mild and did not warrant drug withdrawal.

\section{DISCUSSION}

Hypertension is the most common cardiovascular disease. ${ }^{26}$ Systemic hypertension is one of the most common maladies of mankind affecting about $20 \%$ of population globally. All sections of population in India suffer from the disease with higher prevalence in urban than the rural population. ${ }^{27}$ Hypertension is a key independent risk factor for Cardiovascular Diseases (CVD) such as heart failure, stroke, Coronary Heart Disease (CHD) and end-stage renal disease. However, the treatment of such patients with the antihypertensive agents is able to reduce these complications significantly. During a couple of decades, a number of antihypertensive drugs have been developed and the choice of hypertension treatment has been expanded. ${ }^{7}$

CCBs have been one of the most widely used classes of antihypertensive agents in the last 20 years based on their effectiveness in reducing BP levels, good tolerability and abundant evidence on reducing cardiovascular and renal consequences of hypertension. Dihydropyridine CCBs are now recommended both as first-line therapy and as an ideal partner for dual or triple combination therapies for the clinical management of hypertension and hypertensionrelated comorbidities. ${ }^{12}$

Dihydropyridine-class calcium channel blockers are categorised into three generations according to the length of activity and long-acting calcium channel blockers cause less activation of sympathetic nervous system and are reported to offer beneficial action compared with short-action agents. Novel calcium channel blockers with sustained activity and $\mathrm{T} / \mathrm{N}$-type calcium channel blocking action could exert agentspecific action apart from their class effects, such as the effects on heart rate and renin/aldosterone release, thus providing organ protective effects and may expand the clinical utility of these agents. ${ }^{7}$

The present study aimed at evaluating and comparing the cardiovascular and renoprotective actions of two dihydropyridine - CCBs, amlodipine and cilnidipine.

\section{Effect on Heart Rate}

The mean heart rate was significantly higher in the cilnidipine group compared to the amlodipine group. The mean heart rate in subjects in amlodipine group increased significantly from $74.73 \pm 3.64 \mathrm{bpm}$ at baseline to $77.17 \pm 4$ bpm at the end of 12 weeks. The increase in mean heart rate from baseline was found to be statistically significant at 3 weeks, 6 weeks and 12 weeks after treatment with amlodipine 5 - $10 \mathrm{mg} /$ day. Gender and presence of diabetes mellitus did not significantly affect the increase in heart rate. In a study done by Kaur $\mathrm{M}$ et $\mathrm{al}^{28}$ the pulse rate was found to be significantly higher at the end of 6 weeks in 30 subjects who received amlodipine at a dose of $5-10 \mathrm{mg} /$ day. An increase in daytime pulse rate has also been observed by Hoshide $\mathrm{S}$ et $\mathrm{al}^{29}$ among 55 hypertensive subjects receiving amlodipine $>/-2.5 \mathrm{mg} /$ day.

On the contrary, the mean heart rate of subjects treated with cilnidipine $10 \mathrm{mg} /$ day showed a statistically significant decrease from baseline $(76.63 \pm 3.68 \mathrm{bpm})$ towards the end of 12 weeks $(74.7 \pm 3.55 \mathrm{bpm})$. The decrease was again statistically significant at 3 weeks, 6 weeks and at the end of 12 weeks. No significant difference was observed between either genders and also between diabetic and non-diabetic patients. A similar statistically significant decrease in heart rate has been observed with cilnidipine therapy in a study done by Manthri $\mathrm{S}$ et $\mathrm{al}^{30} \mathrm{~A}$ study by Tanaka $\mathrm{M}$ et $\mathrm{al}^{31}$ has shown a significant decrease in heart rate with cilnidipine therapy in 25 hypertensive subjects also having type 2 diabetes mellitus. Also, a significant decrease in heart rate with cilnidipine therapy has been observed in hypertensive patients with CKD on treatment with an RAS inhibitor in a study done by Hatta T et al.32

Our study also showed a significant difference in the change in mean heart rate from baseline at 6 weeks and 12 weeks between the amlodipine and cilnidipine groups. These results correlate well with the observations made in studies done by Zaman et al, ${ }^{18}$ Kaur $\mathrm{M}$ et $\mathrm{al}^{28}$ and Hoshide $\mathrm{S}$ et al. ${ }^{29}$

Elevated heart rate is associated with higher risk of allcause mortality and cardiovascular events. Ischaemic events, stroke, heart failure and renal failure are the consequences of ignoring sympathetic overactivity in patients with high blood pressure according to $30.91 \%, 25.39 \%, 20.97 \%$ and $22.30 \%$ physicians respectively according to a study done by Dalvi $\mathrm{K}$ et al. ${ }^{33}$ A significant decrease in heart rate with cilnidipine therapy can be attributed to its dual L/N-type calcium channel blocking property. Its inhibitory action on N-type calcium channels decreases norepinephrine release from the nerve terminals, thus explaining its sympatholytic property. ${ }^{19}$ Another study by Sakata et $\mathrm{al}^{14}$ has shown that cilnidipine suppresses cardiac sympathetic overactivity, while amlodipine had little such suppressive effect. Hence, cilnidipine has a significant advantage over pure L-type CCB amlodipine in terms of effect on heart rate, thereby contributing to decreased cardiovascular morbidity and mortality.

\section{Effect on Blood Pressure}

The mean SBP and DBP values in our study were comparable at baseline in both the groups in sitting and standing position.

\section{Sitting BP}

The mean SBP decreased from $127.13 \pm 4.02 \mathrm{mmHg}$ and $127.07 \pm 4.51 \mathrm{mmHg}$ at baseline to $126.33 \pm 4.76 \mathrm{mmHg}$ and $127.07 \pm 4.51 \mathrm{mmHg}$ at the end of 12 weeks in the amlodipine group and cilnidipine group respectively. The mean DBP values too showed a decrease from $78 \pm 5.04$ $\mathrm{mmHg}$ and $78.2 \pm 4.31$ at baseline to $77.53 \pm 3.59 \mathrm{mmHg}$ and $77.8 \pm 3.98 \mathrm{mmHg}$ at the end of the study period in the amlodipine group and cilnidipine group respectively.

\section{Standing BP}

The mean SBP decreased from $122.13 \pm 4.13 \mathrm{mmHg}$ and $121.93 \pm 4.83 \mathrm{mmHg}$ at baseline to $121.33 \pm 4.94 \mathrm{mmHg}$ and $120.93 \pm 3.78 \mathrm{mmHg}$ at the end of 12 weeks in the amlodipine group and cilnidipine group respectively. The mean DBP values also showed a decrease from $74.2 \pm 5.37$ $\mathrm{mmHg}$ and $74.3 \pm 4.16$ at baseline to $73.8 \pm 3.8 \mathrm{mmHg}$ and $73.7 \pm 4.32 \mathrm{mmHg}$ at the end of the study period in the amlodipine group and cilnidipine group respectively. 
The variation in mean SBP and mean DBP was not significant in either of the groups during the 12 weeks study period both in sitting and standing position. Gender and diabetes mellitus did not have any significant influence on the change in any of the BP parameters. However, in a study done by Verma $U$ et $\mathrm{al}^{34}$ there was a statistically significant and dose dependent fall in blood pressure after 2 weeks of treatment with amlodipine, which continued till 12 weeks. Our results also differ from the observations made in studies done by Mion Jr D et $\mathrm{al}^{35}$ and Wilson TW et al,36 where treatment with amlodipine has resulted in a significant fall in both SBP and DBP values among hypertensive patients in the sitting position. While Makawana $\mathrm{Y}$ et $\mathrm{al}^{37}$ have shown a significant reduction in mean blood pressure values with cilnidipine treatment, another study by Manthri $S$ et al $^{30}$ has shown a statistically significant decrease in mean SBP and mean DBP values in hypertensive subjects treated with cilnidipine. Also, in a study done by Hoshide et $\mathrm{al}^{29}$ a significant reduction in clinic as well as ambulatory SBP and DBP values was noted among hypertensive patients treated with either amlodipine or cilnidipine over a period of 16 weeks.

The intergroup difference in terms of mean fall in SBP and DBP values was also not statistically significant at any of the followup visits, both in sitting as well as standing position which implies a similar rate of blood pressure control with both the study medications. These results correlate well with observations made by Zaman ZA et al, ${ }^{18}$ Kaur M et al ${ }^{28}$ Abe M et $\mathrm{al}^{38}$ and Ando K et al. ${ }^{17}$

Calcium channel blockers such as amlodipine and cilnidipine are very effective antihypertensive agents by virtue of their action on L-type calcium channels present in the vascular smooth muscles. The reason for a non-significant change in mean SBP and mean DBP in our study could be attributed to a lack of washout period before initiating treatment with the study medications. As a result, all the subjects in our study had their blood pressure under control with the previous antihypertensive medication, which could have affected the magnitude of fall in SBP and DBP values.

\section{Effect on Proteinuria}

In our study, urine protein/creatinine ratio (UPCR) in a spot urine sample was used to assess proteinuria. In the amlodipine group, the UPCR increased from $0.2773 \pm 0.03$ $\mathrm{mg} / \mathrm{mg}$ at baseline to $0.2817 \pm 0.04 \mathrm{mg} / \mathrm{mg}$ at the end of 12 weeks, which was statistically significant. These results are in concordance with the observations made by Kojima S et al $^{39}$ and Fujita $\mathrm{T}$ et al. ${ }^{40}$ However, a study by Jalal $\mathrm{S}$ et $\mathrm{al}^{41}$ has shown no significant change in urinary albumin excretion rate and another study by Janssen $\mathrm{J} J$ et $\mathrm{al}^{42}$ has shown no significant change in urinary protein excretion with amlodipine therapy. Factors such as daily protein intake and presence of co-existing diabetes mellitus in our study subjects might explain such a difference observed.

In contrast to amlodipine group, subjects in cilnidipine group had a significant decrease in UPCR from $0.28 \pm 0.03$ $\mathrm{mg} / \mathrm{mg}$ at baseline to $0.24 \pm 0.03 \mathrm{mg} / \mathrm{mg}$ at 12 weeks of treatment. A similar significant decrease in proteinuria with cilnidipine therapy has been observed in a study done by Hatta $\mathrm{T}$ et al. ${ }^{32}$ Also, studies by Makawana $\mathrm{Y}$ et $\mathrm{al}^{37}$ and Manthri $S$ et al ${ }^{30}$ have all shown a significant decrease in urinary albumin excretion in hypertensive subjects treated with cilnidipine. Another study by Tsuchihashi $\mathrm{T}$ et $\mathrm{al}^{43}$ has shown that cilnidipine reduces proteinuria in essential hypertension, but not in renal hypertensive patients.

Our study also showed a statistically significant difference in the change in mean UPCR values from baseline to 12 weeks between the amlodipine and cilnidipine groups, which is in concordance with studies done by Zaman ZA et al,18 Abe M et al,38 Uchida $S$ et al,44 Fujita $\mathrm{T}$ et $\mathrm{al}^{40}$ and Kojima $\mathrm{S}$ et al. ${ }^{39}$

A significant decrease in albumin-creatinine ratio among hypertensive patients with type 2 diabetes mellitus was observed in a study done by Tanaka $\mathrm{M}$ et al, ${ }^{31}$ which showed a positive correlation with the change in heart rate indicating cilnidipine may exert its renoprotective effect by inhibiting sympathetic nervous activity. The anti-proteinuric action of cilnidipine can also be attributed to its action on N-type calcium channels present in the kidneys. N-type calcium channels are present in the efferent arterioles and the podocytes. Cilnidipine, by virtue of blocking N-type calcium channels decreases the glomerular pressure, thereby offering significant podocyte protection which contributes to its antiproteinuric effect. 45

\section{Effect on Lipid Profile}

In the present study, the lipid parameters that were evaluated were serum Total Cholesterol (TC), serum Low Density Lipoprotein (LDL), serum High Density Lipoprotein (HDL) and Serum Triglycerides (TG). In the amlodipine group, the mean TC increased from $173.2 \pm 22.24 \mathrm{mg} / \mathrm{dL}$ to $173.93 \pm 18.42 \mathrm{mg} / \mathrm{dL}$ at the end of 12 weeks. The mean LDL and HDL changed from $106.93 \pm 20.91 \mathrm{mg} / \mathrm{dL}$ and $47.6 \pm 5.63$ $\mathrm{mg} / \mathrm{dL}$ at baseline to $106.37 \pm 18.66 \mathrm{mg} / \mathrm{dL}$ and $47.6 \pm 4.97$ $\mathrm{mg} / \mathrm{dL}$ at the end of 12 weeks respectively. Lastly, the mean TG values increased from $116 \pm 57.78 \mathrm{mg} / \mathrm{dL}$ at baseline to $117.10 \pm 53.98 \mathrm{mg} / \mathrm{dL}$ following 12 weeks of treatment. None of the changes observed in the above lipid parameters were statistically significant. These observations correlate well with the observation made by Ahmed $\mathrm{AH}$ et $\mathrm{al}^{46}$ among hypertensive patients treated with amlodipine. Also, a study by Faglia $\mathrm{E}$ et $\mathrm{al}^{47}$ has shown no long-term negative effects on lipid profile on treatment with amlodipine in mild-tomoderate hypertensive subjects also having non-insulin dependent diabetes mellitus.

A few studies, however, have shown slightly different results than those observed in our study. While a study by Amer et $\mathrm{al}^{26}$ has shown a significant reduction in serum LDL and serum TC, studies by Tamuli S et al ${ }^{48}$ and Ahaneku JE et $\mathrm{al}^{49}$ have shown a significant increase in HDL and significant decrease in TG levels respectively with amlodipine therapy. These differences observed might be because the lipid levels of patients also depend on dietary fat intake, physical activity and other lifestyle factors.

The change in mean TC, mean HDL and mean TG did not show any significant difference between diabetic and nondiabetic subjects. However, there was a significant difference between diabetic and non-diabetic subjects treated with amlodipine in terms of change in mean LDL levels $(\mathrm{p}<0.05)$ This beneficial effect of amlodipine on serum LDL levels in non-diabetic subjects compared to diabetic subjects has not been shown in any of the previous studies and needs further studies in a larger group of patients to confirm the findings.

In the cilnidipine group, the mean TC increased from $174.33 \pm 17.73 \mathrm{mg} / \mathrm{dL}$ to $176.77 \pm 16.41 \mathrm{mg} / \mathrm{dL}$ at the end of 
12 weeks. The mean LDL and HDL changed from $108.6 \pm$ $17.41 \mathrm{mg} / \mathrm{dL}$ and $45.57 \pm 5.37 \mathrm{mg} / \mathrm{dL}$ at baseline to $107.67 \pm$ $16.65 \mathrm{mg} / \mathrm{dL}$ and $45.8 \pm 4.42 \mathrm{mg} / \mathrm{dL}$ at the end of 12 weeks respectively. Lastly, the mean TG values increased from $114.57 \pm 47.43 \mathrm{mg} / \mathrm{dL}$ at baseline to $115.63 \pm 43.29 \mathrm{mg} / \mathrm{dL}$ following 12 weeks of treatment. None of the changes observed in the above lipid parameters were statistically significant. Also, no significant difference was observed between the diabetic and non-diabetic subjects treated with cilnidipine with respect to change in any of the lipid parameters assessed in our study. These findings are in concordance with the observations made by Manthri $\mathrm{S}$ et al,30 Ahaneku JE et al,50 Masuda $\mathrm{T}$ et $\mathrm{al}^{51}$ and Zaman ZA et al ${ }^{18}$ among hypertensive subjects irrespective of the diabetic status who were treated with cilnidipine. However, a significant decrease in serum TG levels among hypertensive subjects with concomitant diabetes mellitus as observed by Manthri S et $\mathrm{al}^{30}$ was not seen in our study possibly due to lifestyle differences.

Finally, the change in the mean values of all the lipid parameters assessed in our study was found to be statistically insignificant between the amlodipine group and cilnidipine group. These results correlate well the observations made in studies done by Zaman ZA et al ${ }^{18}$ and Masuda T et al. ${ }^{51}$

\section{Adverse Effects}

The most common adverse effects in both amlodipine and cilnidipine groups were dizziness and headache. This could be because of the hypotensive action of these drugs. Also, while 4 subjects in amlodipine group complained of palpitation, none of the subjects in the cilnidipine group had such complaints. A significant increase in heart rate among subjects in amlodipine group justifies such an observation. In our study, subjects in amlodipine group had a significantly higher incidence of pedal oedema [5 (16.7\%)] compared to the cilnidipine group [1 (3.3\%)]. Similar observation has been made in a study by Adake $P$ et al ${ }^{52}$ among 60 hypertensive patients treated with either amlodipine or cilnidipine. Also, in a study done by Dalvi $\mathrm{K}$ et al,33 many Indian physicians have reported pedal oedema as a common complaint in patients treated with amlodipine, while treatment with cilnidipine minimised pedal oedema. Similarly, in studies done by Shetty $\mathrm{R}$ et $\mathrm{al}^{53}$ and Prasad RS et al ${ }^{54}$ cilnidipine resulted in complete resolution of amlodipine-induced oedema in all the cases without significant worsening of hypertension or tachycardia.

L-type CCBs like amlodipine directly inhibit pre-capillary vasoconstriction through arteriolar dilatation, thus promoting interstitial oedema. However, cilnidipine being a dual L/N-type CCB, dilates both pre- and post-capillary vessels which reduces capillary hypertension, thereby reducing hyperfiltration of fluid into the interstitium. ${ }^{54}$

\section{CONCLUSION}

The present study shows that both amlodipine and cilnidipine are equally effective in terms of blood pressure control in hypertensive patients. The use of amlodipine is associated with reflex tachycardia and a significant increase in the urinary protein excretion rate, which adversely affects the prognosis in hypertensive patients as opposed to cilnidipine, which significantly decreases heart rate and proteinuria.
This beneficial effect of cilnidipine could be attributed to its dual L/N-type calcium channel blocking property unlike amlodipine which is a pure L-type calcium channel blocker. However, both amlodipine and cilnidipine have no significant effect on lipid profile (TC, LDL, HDL and TG).

Hence, we conclude that cilnidipine is a better alternative to amlodipine in hypertensive patients with proteinuria due to its cardioprotective and renoprotective action.

Further long-term studies comparing the two drugs with a larger sample size is warranted to confirm the above findings.

\section{Summary}

This was a randomised, parallel group, open label, singlecentre study comparing the effect of amlodipine and cilnidipine on heart rate, blood pressure, lipid profile and urinary protein excretion among hypertensive subjects with proteinuria. After obtaining Institutional Ethical Committee approval, a total of 60 subjects meeting the inclusion and exclusion criteria were enrolled into the study and randomised to receive either amlodipine $5-10 \mathrm{mg} /$ day or cilnidipine $10-20 \mathrm{mg} /$ day for a period of 12 weeks. The heart rate and blood pressure were monitored at weekly intervals. Lipid profile (TC, LDL, HDL, TG) was measured at baseline, 6 weeks and 12 weeks.

Lastly, the quantitative estimation of proteinuria by urine Protein-to-Creatinine Ratio (UPCR) was done at baseline and 12 weeks. Repeated measure ANOVA was used to analyse the variation in each parameter over the course of 12 weeks. Independent sample ' $\mathrm{t}$ ' test was used to compare the effects of amlodipine and cilnidipine on all parameters. The mean age group, mean BMI and gender distribution was comparable among the two groups. Also, there was no significant difference in the number of diabetics and dyslipidaemic subjects between the two groups. The change in mean SBP and mean DBP was not significant either within each group or between the two groups. While heart rate and UPCR significantly increased at the end of evaluation period in amlodipine group, subjects in cilnidipine group had a significant decrease in heart rate and UPCR, the intergroup difference also being significant. However, neither of the drugs significantly changed any of the lipid parameters assessed in this study. There was a significantly higher incidence of pedal oedema among subjects treated with amlodipine than among those treated with cilnidipine. The above results show that amlodipine and cilnidipine have comparable effects on blood pressure control and lipid profile. However, cilnidipine has a favourable effect on heart rate and urinary protein excretion as opposed to amlodipine, which adversely affects these parameters.

\section{REFERENCES}

[1] Rao CR, Kamath VG, Shetty A, et al. High blood pressure prevalence and significant correlates: a quantitative analysis from coastal Karnataka, India. ISRN Prev Med 2012;2013:574973.

[2] Sutters M. Systemic hypertension. In: Papadakis MA, McPhee SJ, Rabow MW, (eds). Current medical diagnosis and treatment. New York: McGraw-Hill Medical 2013. 
[3] Sarafidis PA, Khosla N, Bakris GL. Antihypertensive therapy in the presence of proteinuria. Am J Kidney Dis 2007;49(1):12-26.

[4] Ogihara T, Kikuchi K, Matsuoka H, et al. The Japanese society of hypertension guidelines for the management of hypertension (JSH 2009). Hypertens Res 2009;32(1):3-107.

[5] Khatib OMN, El-Guindy MS. Clinical guidelines for the management of hypertension. EMRO Technical Publications Series 29. Cairo: WHO; 2005.

[6] Bell K, Twiggs J, Olin BR. Hypertension: the silent killer: updated JNC-8 guideline recommendations. Alabama Pharm Assoc 2015:1-8.

[7] Ozawa Y, Hayashi K, Kobori H. New generation calcium channel blockers in hypertensive treatment Curr Hypertens Rev 2006;2(2):103-11.

[8] Weber MA, Schiffrin EL, White WB, et al. Clinical practice guidelines for the management of hypertension in the community a statement by the American society of hypertension and the international society of hypertension. J Clin Hypertens (Greenwich) 2014;16(1):14-26.

[9] Steffen HM. Amlodipine-a third generation dihydropyridine calcium antagonist. Journal of Clinical and Basic Cardiology 1999;2(1):45-52.

[10] Bergsman JB, Wheeler DB, Tsien RW. Classification and function of voltage-gated calcium channels. $1^{\text {st }}$ edn. In: Endo M, Kurachi Y, Mishina M, (eds). Pharmacology of ionic channel function: activators and inhibitors. Berlin Heidelberg: Springer-Verlag 2000:55-86.

[11] Takahara A. Cilnidipine: a new generation $\mathrm{Ca}^{2+}$ channel blocker with inhibitory action on sympathetic neurotransmitter release. Cardiovasc Ther 2009;27(2):124-39.

[12] Tocci G, Battistoni A, Passerini J, et al. Calcium channel blockers and hypertension. J Cardiovasc Pharmacol Ther 2014;20(2):121-30.

[13] Sica DA. Pharmacotherapy review: calcium channel blockers. J Clin Hypertens (Greenwich) 2006;8(1):536.

[14] Sakata K, Shirotani M, Yoshida H, et al. Effects of amlodipine and cilnidipine on cardiac sympathetic nervous system and neurohormonal status in essential hypertension. Hypertension 1999;33(6):1447-52.

[15] Buffet L, Ricchetti C. Chronic kidney disease and hypertension: a destructive combination. US Pharm 2012;37(6):26-9.

[16] Segura J, Garcia-Donaire JA, Ruilope LM. Calcium channel blockers and renal protection: insights from the latest clinical trials. J Am Soc Nephrol 2005;16(Suppl 1):S64-6.

[17] Ando K, Ueshima K, Tanaka S, et al. Comparison of the antialbuminuric effects of L-/N-type and L-type calcium channel blockers in hypertensive patients with diabetes and microalbuminuria: the study of assessment for kidney function by urinary microalbuminin randomized (SAKURA) trial. Int J Med Sci 2013;10(9):1209-16.

[18] Zaman ZA, Kumari V. Comparison of the effects of amlodipine and cilnidipine on blood pressure, heart rate, proteinuria and lipid profile in hypertensive patients. Int J Basic Clin Pharmacol 2013;2(2):160-4.
[19] Takahara A. Dual L/N-Type Ca $2+$ channel blocker: cilnidipine as a new type of antihypertensive drug. In: Babaei H, (eds). Antihypertensive drugs. Shanghai: InTech; 2012:651-5.

[20] HDL direct. erbalachema.com. 2013 [cited 18 October 2016]. Available from: https://www.erbalachema.com/attachments/HDL\%2 ODIRECT_XSYS0043_0078_B.pdf

[21] LDL direct. erbalachema.com. 2015 [cited 18 October 2016]. Available from: https://www.erbalachema.com/attachments/LDL\%2 0DIRECT_XSYS0080_D.pdf

[22] Triglycerides reagent. thermofisher.com. 2008 [cited 18 October 2016]. Available from: http://tools.thermofisher.com/content/sfs/manuals/ D03103 .pdf

[23] Kupke IR. Enzymatic determination of cholesterol in serum lipoproteins. J Clin Chem Clin Biochem 1976;14(5):217-23.

[24] Nishi HH, Elin RJ. Three turbidimetric methods for determining total protein compared. Clin Chem 1985;31(8):1377-80.

[25] Toora BD, Rajagopal G. Measurement of creatinine by Jaffe's reaction-determination of concentration of sodium hydroxide required for maximum color development in standard, urine and protein free filtrate of serum. Indian J Exp Biol 2002;40(3):352-4.

[26] Amer L, Khurshid R, Farooqi B, et al. To study the effects of amlodipine (Calcium Channel Blocker) on lipid profile in hypertensive patients. Proceeding S.Z.P.G.M.I 2003;17(2):61-6.

[27] Padmavathi T, Ramya JE, Meeanakshi B. A randomized, prospective study to compare the efficacy and tolerability of S-amlodipine $2.5 \mathrm{mg}$ versus racemic amlodipine $5 \mathrm{mg}$ in mild to moderate hypertension. Res Rev J Pharm Toxicol Stud 2014;2(2):26-33.

[28] Kaur M, Sharma AK, Mahajan DS, et al. A comparative study of therapeutic effects and tolerability profile of cilnidipine versus amlodipine in mild to moderate essential hypertension. IJPSR 2012;3(12):5044-55.

[29] Hoshide S, Kario K, Ishikawa J, et al. Comparison of the effects of cilnidipine and amlodipine on ambulatory blood pressure. Hypertens Res 2005;28(12):1003-8.

[30] Manthri S, Veena D, Ambari R. A prospective interventional study on clinical effects of cilnidipine in hypertensive patients. Int J Pharm Tech Res 2015;8(10):70-6.

[31] Tanaka M. The L/N-type calcium channel blocker, cilnidipine, reduces heart rate and albuminuria in patients with type 2 diabetes. J Int Med Res 2010;38(2):602-10.

[32] Hatta T, Takeda K, Shiotsu Y, et al. Switching to an L/N-type calcium channel blocker shows renoprotective effects in patients with chronic kidney disease: the Kyoto cilnidipine study. J Int Med Res 2012;40(4):1417-28.

[33] Dalvi K, Trailokya A, Patel K. Practice of Indian physicians towards use of calcium channel blockers in the management of hypertension: a paper based questionnaire survey. Ind Med Gaz 2015:34-9.

[34] Verma U, Bano G, Lal M, et al. Antihypertensive efficacy of carvedilol and amlodipine in patients of mild to moderate hypertension-a comparative study. JK Sci 2004;6(4):193-6. 
[35] Mion D, Ortega KC, Gomes MA, et al. Amlodipine 2.5mg once daily in older hypertensives: a Brazilian multicentre study. Blood Press Monit 2004;9(2):83-9.

[36] Wilson TW, Lacourciere Y, Barnes CC. The antihypertensive efficacy of losartan and amlodipine assessed with office and ambulatory blood pressure monitoring. CMAJ 1998;159(5):469-76.

[37] Makawana Y, Panchal PJ. Evaluation of efficacy and safety of cilnidipine and losartan in hypertensive patients with type 2 diabetes mellitus. Int J Med Public Health 2016;6(1):40-4.

[38] Abe M, Maruyama N, Suzuki H, et al. L/N-type calcium channel blocker cilnidipine reduces plasma aldosterone, albuminuria, and urinary liver-type fatty acid binding protein in patients with chronic kidney disease. Heart Vessels 2013;28(4):480-9.

[39] Kojima S, Shida M, Yokoyama H. Comparison between cilnidipine and amlodipine besylate with respect to proteinuria in hypertensive patients with renal diseases. Hypertens Res 2004;27(6):379-85.

[40] Fujita T, Ando K, Nishimura H, et al. Antiproteinuric effect of the calcium channel blocker cilnidipine added to renin-angiotensin inhibition in hypertensive patients with chronic renal disease. Kidney Int 2007;72(12):1543-9.

[41] Jalal S, Sofi FA, Abass SM, et al. Effect of amlodipine and lisinopril on microalbuminuria in patients with essential hypertension: a prospective study. Indian J Nephrol 2010;20(1):15-20.

[42] Janssen JJ, Gans RO, van der Meulen J, et al. Comparison between the effects of amlodipine and lisinopril on proteinuria in nondiabetic renal failure-a double-blind, randomized prospective study. Am J Hypertens 1998;11(9):1074-9.

[43] Tsuchihashi $T$, Ueno $M$, Tominaga $M$, et al. Antiproteinuric effect of an N-type calcium channel blocker, cilnidipine. Clin Exp Hypertens 2005;27(8):583-91.

[44] Uchida S, Takahashi M, Sugawara M, et al. Effects of the N/L-type calcium channel blocker cilnidipine on nephropathy and uric acid metabolism in hypertensive patients with chronic kidney disease (J-CIRCLE Study). J Clin Hypertens 2014;16(10):746-53.
[45] Shete MM. Cilnidipine: next generation calcium channel blocker. J Assoc Physicians India 2016;64(4):95-9.

[46] Ahmed AH, Al-Hayali RMA. Effect of amlodipine on serum lipid profile in hypertensive patients. Ann Coll Med 2009;35(1):8-12.

[47] Faglia E, Favales F, Quarantiello A, et al. Effect of amlodipine on insulin secretion, glucose, lipid profile and urinary albumin excretion in patients with mild hypertension and non-insulin dependent diabetes. Clin Drug Investig 1997;13(1):56-66.

[48] Tamuli S, Kakati S, Das S, et al. Comparative studies of efficacy and effects on oxidative stress of amlodipine and ramipril in the hypertensive patients of north east India. Int J Pharm Pharm Sci 2015;7(12):118-21.

[49] Joseph E, Sakata K, Urano T, et al. Lipids, lipoproteins and fibrinolytic parameters during amlodipine treatment of hypertension. Journal of Health Science 2000;46(6):455-8.

[50] Ahaneku JE, Sakata K, Uranol T, et al. Effects of cilnidipine on lipids, lipoproteins and fibrinolytic system in hypertensive patients. Drugs Exp Clin Res 2000;26(4):119-23.

[51] Kanaoka T, Tamura K, Wakui H, et al. L/N-type calcium channel blocker cilnidipine added to renin-angiotensin inhibition improves ambulatory blood pressure profile and suppresses cardiac hypertrophy in hypertension with chronic kidney disease. Int $\mathrm{J}$ Mol Sci 2013;14(8):16866-81.

[52] Adake P, Somashekar HS, Rafeeq MPK, et al. Comparison of amlodipine with cilnidipine on antihypertensive efficacy and incidence of pedal edema in mild to moderate hypertensive individuals: a prospective study. J Adv Pharm Technol Res 2015;6(2):81-5.

[53] Shetty R, Vivek G, Naha K, et al. Excellent tolerance to cilnidipine in hypertensives with amlodipine induced edema. N Am J Med Sci 2013;5(1):47-50.

[54] Prasad RS. Replacement of amlodipine with cilnidipine and assessment of pedal edema along with blood pressure control. Sch J App Med Sci 2015;3 (4A): 1680-2. 